This is the post print version of the article, which has been published in Blood pressure. 2019, 28 (1),4-14.https://doi.org/10.1080/08037051.2018.1521263.

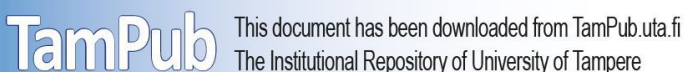

\title{
LDL cholesterol is associated with systemic vascular resistance and wave reflection in subjects naive to cardiovascular drugs
}

\author{
Manoj Kumar Choudhary ${ }^{\mathrm{a}}$, Arttu Eräranta ${ }^{\mathrm{a}}$, Antti J. Tikkakoski ${ }^{\mathrm{a}, \mathrm{b}}$, Jenni Koskela ${ }^{\mathrm{a}}$, \\ Elina J. Hautaniemi ${ }^{\mathrm{a}}$, Mika Kähönen ${ }^{\mathrm{a}, \mathrm{b}}$, Jukka Mustonen $^{\mathrm{a}, \mathrm{c}}$, Ilkka Pörstia ${ }^{\mathrm{a}, \mathrm{c}}$
}

\begin{abstract}
${ }^{a}$ Faculty of Medicine and Life Sciences, University of Tampere, Tampere; Finland
${ }^{b}$ Department of Clinical Physiology, Tampere University Hospital, Tampere; Finland

${ }^{c}$ Department of Internal Medicine, Tampere University Hospital, Tampere, Finland.
\end{abstract}

Correspondence to Ilkka Pörsti, M.D., Faculty of Medicine and Life Sciences, P.O. Box 100, FIN-33014 University of Tampere, Finland. Tel. +358 50 4389440; Fax +358 3 3641472; email: ilkka.porsti@uta.fi 


\begin{abstract}
Background and aim: Low density lipoprotein cholesterol (LDL-C) is a primary risk factor for atherosclerosis, but it is also associated with elevated blood pressure (BP) and future development of hypertension. We examined the relationship between LDL-C and haemodynamic variables in normotensive and never-treated hypertensive subjects.
\end{abstract}

Methods: We recruited 615 volunteers (19-72 years) without lipid-lowering and BP-lowering medication. Supine haemodynamics were recorded using continuous radial pulse wave analysis, wholebody impedance cardiography, and single channel electrocardiogram. The haemodynamic relations of LDL-C were examined using linear regression analyses with age, sex, body mass index (BMI) (or height and weight as appropriate), smoking status, alcohol use, and plasma C-reactive protein, sodium, uric acid, high density lipoprotein cholesterol (HDL-C), triglycerides, estimated glomerular filtration rate, and quantitative insulin sensitivity check index as the other included variables.

Results: The mean (SD) characteristics of the subjects were: age 45 (12) years, BMI 27 (4) kg/m², office BP 141/89 (21/13) mmHg, creatinine 74 (14) $\mu \mathrm{mol} / \mathrm{l}$, total cholesterol 5.2 (1.0), LDL-C 3.1 (0.6), triglycerides $1.2(0.8)$, and HDL-C $1.6(0.4) \mathrm{mmol} / \mathrm{l}$. LDL-C was an independent explanatory factor for aortic systolic and diastolic BP, augmentation index, pulse wave velocity (PWV), and systemic vascular resistance index $(p<0.05$ for all). When central BP was included in the model for PWV, LDL$\mathrm{C}$ was no longer an explanatory factor for PWV.

Conclusions: LDL-C is independently associated with BP via systemic vascular resistance and wave reflection. These results suggest that LDL-C may play a role in the pathogenesis of primary hypertension.

Keywords: augmentation index, haemodynamics, hypertension, impedance cardiography, LDL cholesterol, pulse wave analysis, systemic vascular resistance 


\section{Introduction}

Cardiovascular diseases are the leading cause of mortality worldwide representing $31 \%$ of all global deaths [1]. One fourth of all deaths in the Western countries are related to coronary heart disease [2]. Subjects with familial hypercholesterolemia are characterized by premature atherosclerosis, and the pathogenic role of low density lipoprotein cholesterol (LDL-C) in this process is well recognized [3]. LDL-C has also vasoconstrictor, pro-inflammatory and thrombogenic properties, and it functions as a mitogenic factor that can stimulate vascular hypertrophy via several growth factors [4].

Previous studies have reported that LDL-C is associated with arterial stiffness, shown as increased pulse wave velocity (PWV) [5], or reduced aortic compliance using ultrasound measurements [6,7]. A positive correlation between LDL-C and PWV was also observed in a group of 315 children aged 8-9 years [8]. However, according to a systematic review, less than $10 \%$ of studies demonstrated a positive correlation between serum lipids and arterial stiffness, and therefore the influence of risk factors other than age and blood pressure (BP) on PWV appears to be small [9].

Dyslipidaemias are not only a risk factor for cardiovascular disease, but can also predict the future development of hypertension [10-13] and impairment of endothelial function [14]. LDL-C can reduce nitric oxide bioavailability and blunt the vasodilator response to acetylcholine [4,14-17], and the resulting endothelial dysfunction could be manifested as increased BP. Following treatment with statins, several trials have shown moderate but statistically significant lowering effect on BP [18-23], reduction of arterial stiffness [19,20,24], and improved endothelial function [25,26].

Some previous studies that addressed the association of LDL-C with haemodynamic variables included subjects taking antihypertensive medications and even diabetic patients [5-7]. The objective of this cross-sectional study was to investigate the association of LDL-C with haemodynamic variables that 
could potentially explain differences in BP between normotensive subjects and never-treated patients with primary hypertension.

\section{Methods}

\section{Participants}

All subjects participated in an ongoing study with the aim to examine haemodynamics in primary and secondary hypertension versus normotension (DYNAMIC study; ClinicalTrails.gov identifier NCT01742702). The participant recruitment has been described before [27,28], and the study flowchart is presented in Supplementary Figure 1. Subjects taking lipid-lowering or BP-lowering medication or with a history of coronary artery disease, stroke, heart failure, valvular heart disease, diabetes, chronic kidney disease, secondary hypertension, alcohol or substance abuse, psychiatric illnesses, or heart rhythm other than sinus were excluded (total number of enrolled subjects 1349). All subjects underwent physical examination by a medical doctor, measurement of office BP, and routine laboratory analyses for elevated BP according to the guidelines of the European Society of Hypertension [29]. The medical history, lifestyle habits and use of medicines, dietary supplements, and other substances not registered as drugs were documented along with information about smoking and alcohol consumption as standard drinks ( 12 grams of absolute alcohol) per week.

A total of 615 normotensive and never-treated subjects with primary hypertension, aged 19-72 years, were included in the study. Based on the office BP measurements on a single occasion, $249(40.5 \%)$ of the participants were normotensive and 366 (59.5\%) were hypertensive. For the graphical illustrations, they were divided into age- and sex-adjusted LDL-C quartiles (Q1, n=153; Q2, n=158; Q3, n=148; Q4, $\mathrm{n}=156$ ). Signed informed consent was obtained from all participants. The study complies with the 
declaration of Helsinki, and was approved by the ethics committee of the Tampere University Hospital (study code R06086M) and the Finnish Medicines Agency (Eudra-CT registration number 2006002065-39).

Altogether $230(37.4 \%)$ of the 615 persons used some medications. Seventy-six females were treated with systemic oestrogen, progestin, or their combination (contraception, hormone replacement therapy), and 1 subject with tibolone. Forty-one subjects were treated with antidepressants, 18 with antihistamines, 17 with inhaled corticosteroids, 13 with proton pump inhibitors, while 22 euthyroid subjects were on a stable dose of thyroid hormone. Other medications in use were hypnotics or sedatives (8), low dose acetylsalicylic acid (6), non-steroidal anti-inflammatory drugs (4), antirheumatic agents (4), antiepileptics (3), allopurinol (3), coxibs (3), antipsychotics (2), muscle relaxants (2), varenicline (2), antiviral agents (2), paracetamol (1), carbimazole (1), isotretinoin (1), and alendronate (1). One subject was treated with warfarin because of an anti-phospholipid syndrome, and she was physically well and symptomless during the recordings.

\section{Laboratory analyses}

Blood and urine sampling was preceded by about 12 hours of fasting. Plasma total, high density lipoprotein cholesterol (HDL-C), LDL-C, triglyceride, C-reactive protein (CRP), sodium, potassium, glucose, cystatin-C, and creatinine concentrations were determined using Cobas Integra 700/800 (F. Hoffmann-Laroche Ltd, Basel; Switzerland) or Cobas6000, module c501 (Roche Diagnostics, Basel, Switzerland), insulin using electrochemiluminescence immunoassay (Cobas e411, Roche Diagnostics), and blood cell count by ADVIA 120 or 2120 (Bayer Health Care, Tarrytown, NY, USA). Urine dipstick analysis was made by an automated refractometer test (Siemens Clinitec Atlas or Advantus, 
Siemens Healthcare GmbH, Erlangen, Germany). For evaluation of insulin sensitivity the quantitative insulin sensitivity check index (QUICKI) [30] was calculated, and glomerular filtration rate (eGFR) was estimated using the CKD-EPI cystatin C formula [31].

\section{Pulse wave analysis}

Radial BP and pulse wave were continuously recorded from radial pulsation using a tonometric sensor (Colin BP-508T, Colin Medical Instruments Corp., USA), secured on the radial pulse with a wrist band. The radial BP signal was calibrated twice during each 5 minute-period by contralateral brachial BP measurements. Aortic BP was derived with the SphygmoCor system (SpygmoCor PWMx ${ }^{\circledR}$, AtCor medical, Australia) by means of the validated generalized transfer function [32]. Left ventricular ejection duration, aortic pulse pressure and reflection time, augmentation index (AIx, augmented pressure/pulse pressure*100), AIx adjusted to heart rate 75/min (AIx@75), and amplification of pulse pressure and systolic pressure (radial pressure/aortic pressure) were determined [27,28].

\section{Whole-body impedance cardiography}

Beat-to-beat heart rate, stroke volume, cardiac output, and PWV were recorded using whole-body impedance cardiography (CircMon ${ }^{\circledR}$, JR Medical Ltd., Tallinn, Estonia) that detects changes in body electrical impedance during cardiac cycles. The method and electrode configuration have been previously reported [33]. Systemic vascular resistance (SVR) was calculated from the tonometric BP signal and cardiac index measured by $\operatorname{CircMon}^{\circledR}$. SVR was calculated by subtracting normal central venous pressure $(4 \mathrm{mmHg})$ from mean arterial pressure and dividing it by cardiac output. SVR and cardiac output were presented as indexes related to body surface area - cardiac index, and systemic 
vascular resistance index (SVRI), respectively. The stroke volume values measured using $\operatorname{CircMon}^{\circledR}$ correlate well with 3 dimensional ultrasound [34]. The supine cardiac output values measured with CircMon $^{\circledR}$ correlate well with the values measured using thermodilution [33]. The whole-body impedance cardiography tends to overestimate PWV, and a validated equation was utilized to calculate values correspond to the ultrasound method (PWV $=$ PWVimpedance * $0.696+0.864)$ [35]. By the use of this equation, the PWV values recorded using CircMon ${ }^{\circledR}$ show very good correlations with values measured using either ultrasound $(r=0.91)$ [35] or the tonometric SphygmoCor ${ }^{\circledR}$ method $(r=0.82$, bias $0.02 \mathrm{~m} / \mathrm{s}, 95 \%$ confidence interval -0.21 to 0.25$)$ [28].

\section{Experimental protocol}

Haemodynamics were recorded in a quiet, temperature-controlled laboratory by research nurses [36]. Caffeine containing products, smoking or heavy meals were to be avoided for $\geq 4$ hours, and alcohol consumption for $>24$ hours prior to the studies. The subjects rested supine, the left arm with the tonometric sensor abducted to 90 degrees in an arm support. After getting accustomed to the laboratory for about 10 minutes, supine haemodynamics were recorded for five minutes. For the statistical analyses the mean values of each 1-minute period of recording were calculated. The good repeatability and reproducibility of the measurement protocol has been demonstrated [37].

\section{Statistics}

Continuous variables were expressed as the mean and standard deviation (SD) or standard error of the mean (SEM). Baseline characteristics were depicted as age- and sex-adjusted quartiles of LDL-C (Table 1). The demographic and laboratory data was analysed using analysis of variance (ANOVA), 
and the Bonferroni correction was applied in the post-hoc analyses. The homogeneity of variances was tested with the Levene's test. For the illustrations, the haemodynamic differences between the quartiles were examined using ANOVA for repeated measures adjusted for age and sex. Spearman's correlations (rS) were calculated, as appropriate.

A multiple regression analysis with stepwise elimination was applied to evaluate the associations between age, sex, body mass index (BMI) (for systemic vascular resistance index BMI was replaced by height and weight), smoking status, alcohol consumption, QUICKI, plasma CRP, sodium, uric acid, HDL-C, LDL-C, triglycerides, and eGFR (independent variables), and aortic systolic and diastolic BP, aortic pulse pressure, AIx, PWV and SVRI (dependent variables) in model 1. The variables in model 2 were model $1+$ PWV (independent variables) for aortic systolic and diastolic BP, aortic pulse pressure (dependent variables); for AIx (dependent variable): model $1+$ heart rate, SVRI, and PWV (independent variables); for PWV (dependent variable): model $1+$ aortic systolic BP (independent variables). For these analyses the skewed distribution of PWV was corrected by $\lg _{10}$-transformation. $P<0.05$ was considered statistically significant. SPSS version 22.0 (IBM SPSS Statistics, Armonk, NY, USA) was used for the statistics.

\section{Results}

Study population and laboratory values

In total, 615 subjects were included in the analyses, consisting of 314 male (51\%) and 301 female (49\%) subjects (Table 1). The age range was 19-72 years, mean (SD) age was 44.9 (11.9) years, BMI 26.8 (4.4) kg/m², office systolic/diastolic BP 140.5 (21.1) / 89.3 (12.5) mmHg, creatinine 73.7 (13.5) $\mu \mathrm{mol} / \mathrm{l}$, total cholesterol 5.2 (1.0), LDL-C 3.1 (0.6), triglycerides 1.2 (0.8) and HDL-C $1.6(0.4) \mathrm{mmol} / \mathrm{l}$ 
(Table 1). Altogether 80 (13\%) of the subjects had impaired fasting plasma glucose (6.1-7.0 mmol/l), while in 6 subjects $(1 \%)$ the fasting plasma glucose was in the range of $7.1-10.3 \mathrm{mmol} / \mathrm{l}$. However, none of the study participants had glucosuria or proteinuria in the morning urine sample.

For the graphical illustrations the participants were divided into age- and sex-adjusted LDL-C quartiles. The average LDL-C in the quartiles ranged from 2.05 (0.51) (Q1) to $4.15(0.75)(\mathrm{Q} 4) \mathrm{mmol} / \mathrm{l}$ (Table 1). After adjustments for age and sex, the quartiles presented with differences in BMI, office systolic and diastolic BP, eGFR, QUICKI, and plasma cystatin C, total cholesterol, triglycerides, HDL-C, and glucose concentrations (Table 1). Alcohol intake, smoking status, and plasma creatinine, sodium, potassium, uric acid, CRP, and insulin concentrations were not significantly different between the quartiles.

\section{Haemodynamic variables in the quartiles of $L D L-C$ adjusted for age and sex}

Aortic and radial systolic and diastolic BP were not significantly different in adjacent quartiles, but were different in all other comparisons between the quartiles, so that the highest LDL-C quartile (Q4) presented with the highest BP (Figures 1A-1B, Supplementary Figures 2A-2B). Aortic pulse pressure differed between the highest (Q4) and lowest LDL-C quartile (Q1) (Figure 1C), while AIx (Figure 1D) and AIx@75 (Supplementary Figure 2C), and heart rate and cardiac index (Figures 2A-2B) were not significantly different between the quartiles. SVRI differed between Q4 and Q1 (Figure 2C), while PWV was higher in Q4 than in Q1 and Q2 (Figure 2D).

\section{LDL-C and haemodynamic variables in stepwise linear regression analyses}

We performed linear regression analyses to examine the relationships between the haemodynamic variables and age, sex, BMI (for systemic vascular resistance index replaced by height and weight), 
smoking status, weekly alcohol consumption, insulin sensitivity (QUICKI) [30], cystatin C based eGFR [31], and plasma CRP, sodium, uric acid, HDL-C, LDL-C, and triglyceride concentrations (model 1, Table 2). These analyses showed that LDL-C was a significant independent explanatory factor for aortic systolic and diastolic BP, aortic pulse pressure, AIx, PWV, and SVRI ( $p<0.05$ for all) (model 1, Table 2).

The model 2 for aortic systolic and diastolic BP, and aortic pulse pressure included PWV in addition to the above variables of model 1 (model 2, Table 2). These results showed that LDL-C was a significant independent explanatory factor for aortic systolic and diastolic $\mathrm{BP}(p<0.05$ for all), while in this model LDL-C was no more an explanatory factor for aortic pulse pressure.

The model 2 for AIx contained the variables heart rate, SVRI and PWV in addition to the above variables of model 1 . The outcome was that LDL-C, smoking status, sex, age, BMI, heart rate, and SVRI were independent significant explanatory factors for AIx (model 2, Table 2).

The model 2 for PWV contained aortic systolic BP in addition to the variables of model 1. In this model LDL-C was no more an explanatory factor for PWV. The significant explanatory factors for PWV were age, aortic systolic BP, uric acid, triglycerides, HDL-C, smoking status and sodium (model 2, Table 2). If aortic mean BP or aortic diastolic BP was used in the model 2 instead of aortic systolic BP, LDL-C was not an independent explanatory factor for PWV, either (not shown).

\section{Discussion}

LDL-C is an established risk factor for atherosclerosis and endothelial dysfunction, but it has also been linked with elevated BP $[10,11,18,20]$. We examined the relations of LDL-C with several cardiovascular variables using non-invasive recordings of haemodynamics. The present results indicated that LDL-C was independently associated with BP, AIx, and SVRI. LDL-C was also 
associated with increased PWV, but the relation between PWV and LDL-C was no longer significant when central BP was included in the model.

Increased large artery stiffness, manifested as accelerated PWV and elevated pulse pressure, increases with aging and is an independent cardiovascular risk factor [38]. As atherosclerosis and plaque formation alter the properties of the arterial wall, the measures of arterial stiffness have been considered as surrogate markers of atherosclerosis [9,39]. Due to the central role of LDL-C in atherosclerosis, its relation with arterial stiffness would seem evident, and a positive correlation between LDL-C and PWV has been reported [5-8]. However, the relationship between LDL-C and arterial stiffness, a process characterized by increased fibrosis and collagen deposition in the arterial wall, remains controversial: according to a comprehensive review, the majority of studies did not find a positive correlation between serum lipids and arterial stiffness, as measured using determinations of PWV [9].

In addition to age, PWV is strongly dependent on the prevailing level of BP $[9,39]$. In our study LDL-C was associated with PWV, but when central BP was included in the regression model, LDL-C was no more an explanatory factor for arterial stiffness. Our results indicated that triglyceride concentration was directly, and HDL-C was inversely, associated with PWV in the linear regression model 2. Previously, higher triglyceride and lower HDL-C levels have been associated with increased PWV $[40,41]$. Of note, the characteristic components of the metabolic syndrome, i.e. lower HDL-C, higher triglycerides, and higher uric acid were all associated with higher PWV in the present statistical model 2, corresponding to previous findings $[27,40]$. The influence of glucose metabolism on haemodynamics was taken into consideration in the regression analyses by the inclusion of the QUICKI index in the variables. Many reports support the view that statins can improve arterial stiffness $[19,20,24]$. The present results raise the possibility that the lowering effect of statins on PWV could partially be mediated via the beneficial effect on central BP. 
LDL-C may be a significant risk factor for the development of hypertension. Laaksonen et al. found that abnormal LDL-C and triglyceride metabolism predicted future development of hypertension in middle-aged men [11]. In a study comprising 20,074 subjects, the incidence of new onset of hypertension was lower in subjects with lower LDL-C [13]. Several studies have revealed small but significant reductions in BP after treatment with statins [18,19,22,23]. A meta-analysis comprising 828 subjects reported a decrease of $\mathrm{BP}$ by $1.9 / 0.9 \mathrm{mmHg}$ following statin therapy that was unrelated to age, changes in serum cholesterol, or length of the trial [21]. Another meta-analysis including 22,602 statintreated patients and 22,511 controls found that statins decreased BP by 2.62/0.94 $\mathrm{mmHg}$, an effect that was not related to patient age, follow-up duration, or the evaluated quality of the study [22]. In the Anglo-Scandinavian Cardiac Outcomes Trial, 10,305 hypertensive patients were randomly assigned to receive atorvastatin $10 \mathrm{mg}$ daily or placebo for a median follow-up of 3.3 years [42]. Office BP throughout the trial was similar in the atorvastatin and placebo groups. However, no conclusions could be drawn about the lack of BP-lowering effect of atorvastatin in this study, as antihypertensive medication was titrated upwards based on achieved BP, and this potentially masked any impact of atorvastatin on BP [42].

Endothelial dysfunction can be manifested as elevated BP [16], and factors like dyslipidaemia that impair endothelium-dependent vasomotion may play a role in the pathogenesis of primary hypertension $[12,14]$. Blood lipids, including LDL-C, have a number of non-atheromatous effects on blood vessels, which increase oxidative stress and inflammation, and promote elastin damage and deposition of calcium within the arterial wall $[43,44]$. LDL-C has been found to impair endothelial nitric oxide bioavailability through increased vascular production of reactive oxygen species and enhanced responses to vasoconstrictors like angiotensin II $[15,17,45,46]$. Therefore, the consequence of impaired endothelium-mediated dilatation in the resistance vessels would be the elevation of SVR. Twelve month treatment with statins improved flow-mediated dilatation in the brachial artery [25], while a 
meta-analysis reported increased flow-mediated dilatation following treatment with pitavastatin [26]. As the deleterious effect of LDL-C on endothelial dysfunction can be reversed by statins, this provides a potential explanation for the beneficial effects of these agents on BP [20,24-26]. Experimental evidence suggests that LDL-C can increase the angiotensin II type 1 receptor density in the arterial wall $[15,17]$, while statin treatment in vivo can reduce the vasoconstrictor responses elicited by angiotensin II in isolated human internal thoracic artery segments in vitro [47]. Collectively, the above findings support the view that LDL-C is a significant explanatory factor for BP via increased SVR. Supporting this view, plasma total cholesterol was recently found to be independently associated with the media:lumen ratio of small arteries obtained from humans by biopsy [48]. This variable that characterises resistance vessel structure is directly linked to the regulation of SVR [49].

The present results indicated that LDL-C was an independent determinant of AIx (models 1 and 2) and aortic pulse pressure (model 1), corresponding to previous findings [50,51]. AIx and central pulse pressure were higher in subjects with hypercholesterolemia than in controls [50]. Men with higher LDL-C level had increased AIx in all age groups, and a similar finding was observed in women under 60 years of age [51]. A significant proportion of the reflected pressure wave originates from resistance arteries [49], and the level of augmentation is equally influenced by SVR and arterial stiffness [28]. Therefore, the association between LDL-C and wave reflection can be explained via SVR, the lowering of which reduces the magnitude of AIx. We also found an inverse relationship between smoking and SVR, which could be mediated via the vasodilating influence of carbon monoxide in tobacco smoke [52]. Unexpectedly, smoking showed a small inverse association with PWV, and we can speculate that lower SVR may also favour reductions in PWV. Of note, according to a comprehensive review, smoking has not influenced PWV in the majority of studies [9].

The current study has limitations and the interpretation of the results must be done cautiously. Although the methods have been validated against invasive measurements, 3 dimensional ultrasound, 
and tonometric recordings of PWV [28,32-34], the non-invasive evaluation of stroke volume and cardiac output is based on mathematical processing of the bioimpedance signal and simplification of physiology [33]. The supine recordings lasted 5 minutes, and this gives a rather narrow window of observation for the study of haemodynamics. However, when compared with single measurements of BP and heart rate, these continuous evaluations were still based on variables collected from more than 300 cardiac cycles. The cross-sectional design does not allow conclusions about causal relationship, and the present findings should be confirmed in follow-up studies. As the haemodynamic recordings were performed in subjects who were themselves willing to participate, this makes a potential source for selections bias. Although the results were adjusted for multiple covariates that may be associated with LDL-C, the possibility of residual confounding remains. Finally, LDL-C and haemodynamics were measured during one single occasion, and repeated measurements of all variables would strengthen the findings.

In conclusion, the present results showed that LDL-C was independently associated with $\mathrm{BP}$ and this effect could be attributed to elevated SVR that also resulted in enhanced wave reflection. Therefore, LDL-C could play a role in the pathogenesis of primary hypertension, possibly via its harmful influence on endothelium-dependent vasodilation.

\section{Acknowledgements}

The authors are deeply grateful to Paula Erkkilä, research nurse (RN), and Reeta Kulmala, RN, for invaluable technical assistance. 


\section{Disclosure statement}

The authors declare no conflict of interest with respect to this manuscript.

\section{Financial support}

This work was financially supported by Aarne Koskelo Foundation, Competitive State Research Financing of the Expert Responsibility Area of Tampere University Hospital, Finnish Foundation for Cardiovascular Research, Paavo Nurmi Foundation, Päivikki and Sakari Sohlberg Foundation, Sigrid Jusélius Foundation, and Pirkanmaa Regional Fund of the Finnish Cultural Foundation.

\section{Data availability}

Analyses and generated datasets that support the current study are not available publicly. The datasets are available from the corresponding author on reasonable request. 


\section{References}

[1] World health organisation. WHO | Cardiovascular diseases (CVDs). WHO. 2017. Available from: http://www.who.int/mediacentre/factsheets/fs317/en/.

[2] Heron M. Deaths: leading causes for 2010. Natl. Vital Stat. 2013;62:1-96.

[3] Ershova AI, Meshkov AN, Rozhkova TA, et al. Carotid and aortic stiffness in patients with heterozygous familial hypercholesterolemia. PloS One. 2016;11:e0158964.

[4] Rosendorff C. Effects of LDL cholesterol on vascular function. J. Hum. Hypertens. 2002;16:S26-S28.

[5] Wang F, Ye P, Luo L, et al. Association of serum lipids with arterial stiffness in a populationbased study in Beijing. Eur. J. Clin. Invest. 2011;41:929-936.

[6] Dart AM, Lacombe F, Yeoh JK, et al. Aortic distensibility in patients with isolated hypercholesterolaemia, coronary artery disease, or cardiac transplant. The Lancet. 1991;338:270-273.

[7] Giannattasio C, Mangoni AA, Failla M, et al. Combined effects of hypertension and hypercholesterolemia on radial artery function. Hypertension. 1997;29:583-586.

[8] Correia-Costa A, Correia-Costa L, Caldas Afonso A, et al. Determinants of carotid-femoral pulse wave velocity in prepubertal children. Int. J. Cardiol. 2016;218:37-42.

[9] Cecelja M, Chowienczyk P. Dissociation of aortic pulse wave velocity with risk factors for cardiovascular disease other than hypertension: a systematic review. Hypertension. 2009;54:1328-1336.

[10] Halperin RO, Sesso HD, Ma J, et al. Dyslipidemia and the risk of incident hypertension in men. Hypertension. 2006;47:45-50.

[11] Laaksonen DE, Niskanen L, Nyyssonen K, et al. Dyslipidaemia as a predictor of hypertension in middle-aged men. Eur. Heart J. 2008;29:2561-2568.

[12] Oparil S, Zaman MA, Calhoun DA. Pathogenesis of hypertension. Ann. Intern. Med. 2003;139:761-776.

[13] Borghi C, Cicero AFG, Saragoni S, et al. Rate of control of LDL cholesterol and incident hypertension requiring antihypertensive treatment in hypercholesterolemic subjects in daily clinical practice. Ann. Med. 2014;46:97-102.

[14] Rajendran P, Rengarajan T, Thangavel J, et al. The Vascular endothelium and human diseases. Int. J. Biol. Sci. 2013;9:1057-1069.

[15] Nickenig G, Sachinidis A, Michaelsen F, et al. Upregulation of vascular angiotensin II receptor gene expression by low-density lipoprotein in vascular smooth muscle cells. Circulation. 1997;95:473-478. 
[16] Fleming I. Nitric Oxide: Biology and Pathobiology. Third Ed. Academic Press; 2017. Chapter 23 - NO signaling defects in hypertension; p. 301-311.

[17] Nickenig G. Central role of the AT1-receptor in atherosclerosis. J. Hum. Hypertens. 2002;16:S26-S33.

[18] Lamarche F, Agharazii M, Nadeau-Fredette A-C, et al. Central and brachial blood pressures, statins, and low-density lipoprotein cholesterol: a mediation analysis. Hypertension. 2018;71:415-421.

[19] Oh PC, Han SH, Koh KK, et al. Rosuvastatin treatment improves arterial stiffness with lowering blood pressure in healthy hypercholesterolemic patients. Int. J. Cardiol. 2014;176:1284-1287.

[20] Canepa M, Artom N, Ameri P, et al. Short-term effect of rosuvastatin treatment on arterial stiffness in individuals with newly-diagnosed heterozygous familial hypercholesterolemia. Int. J. Cardiol. 2018;255:215-220.

[21] Strazzullo P, Kerry SM, Barbato A, et al. Do statins reduce blood pressure? A meta-analysis of randomized, controlled trials. Hypertension. 2007;49:792-798.

[22] Briasoulis A, Agarwal V, Valachis A, et al. Antihypertensive effects of statins: a meta-analysis of prospective controlled studies. J. Clin. Hypertens. 2013;15:310-320.

[23] Tocci G, Presta V, Citoni B, et al. Favourable impact of statin use on diastolic blood pressure levels: analysis of a large database of 24-hour ambulatory blood pressure monitoring. J. Hypertens. 2017;35:2086-2094.

[24] Upala S, Wirunsawanya K, Jaruvongvanich V, et al. Effects of statin therapy on arterial stiffness: a systematic review and meta-analysis of randomized controlled trial. Int. J. Cardiol. 2017;227:338-341.

[25] Demir V, Doğru MT, Ede H, et al. The effects of treatment with atorvastatin versus rosuvastatin on endothelial dysfunction in patients with hyperlipidaemia. Cardiovasc. J. Afr. 2018;29:1-5.

[26] Katsiki N, Reiner Ž, Reiner ET, et al. Improvement of endothelial function by pitavastatin: a meta-analysis. Expert Opin. Pharmacother. 2018;19:279-286.

[27] Kangas P, Tikkakoski AJ, Tahvanainen AM, et al. Metabolic syndrome may be associated with increased arterial stiffness even in the absence of hypertension: A study in 84 cases and 82 controls. Metabolism. 2013;62:1114-1122.

[28] Wilenius M, Tikkakoski AJ, Tahvanainen AM, et al. Central wave reflection is associated with peripheral arterial resistance in addition to arterial stiffness in subjects without antihypertensive medication. BMC Cardiovasc. Disord. 2016;16:131.

[29] Mancia G, Fagard R, Narkiewicz K, et al. 2013 ESH/ESC Guidelines for the management of arterial hypertension. The task force for the management of arterial hypertension of the European society of hypertension (ESH) and of the European society of cardiology (ESC). Eur. Heart J. 2013;34:2159-2219. 
[30] Katz A, Nambi SS, Mather K, et al. Quantitative insulin sensitivity check index: a simple, accurate method for assessing insulin sensitivity in humans. J. Clin. Endocrinol. Metab. 2000;85:2402-2410.

[31] Inker LA, Schmid CH, Tighiouart H, et al. Estimating glomerular filtration rate from serum creatinine and cystatin C. N. Engl. J. Med. 2012;367:20-29.

[32] Chen C-H, Nevo E, Fetics B, et al. Estimation of central aortic pressure waveform by mathematical transformation of radial tonometry pressure: validation of generalized transfer function. Circulation. 1997;95:1827-1836.

[33] Kööbi T, Kaukinen S, Ahola T, et al. Non-invasive measurement of cardiac output: whole-body impedance cardiography in simultaneous comparison with thermodilution and direct oxygen fick methods. Intensive Care Med. 1997;23:1132-1137.

[34] Koskela JK, Tahvanainen A, Haring A, et al. Association of resting heart rate with cardiovascular function: a cross-sectional study in 522 Finnish subjects. BMC Cardiovasc. Disord. 2013;13:102.

[35] Kööbi T, Kähönen M, Iivainen T, et al. Simultaneous non-invasive assessment of arterial stiffness and haemodynamics-a validation study. Clin. Physiol. Funct. Imaging. 2003;23:31-36.

[36] Tikkakoski AJ, Tahvanainen AM, Leskinen MH, et al. Hemodynamic alterations in hypertensive patients at rest and during passive head-up tilt. J. Hypertens. 2013;31:906.

[37] Tahvanainen A, Koskela J, Tikkakoski A, et al. Analysis of cardiovascular responses to passive head-up tilt using continuous pulse wave analysis and impedance cardiography. Scand. J. Clin. Lab. Invest. 2009;69:128-137.

[38] Benetos A, Alecu C, Aubry C, et al. Determinants of arterial stiffness in an apparently healthy population over 60 years. J. Hum. Hypertens. 2006;20:749.

[39] Laurent S, Cockcroft J, Van Bortel L, et al. Expert consensus document on arterial stiffness: methodological issues and clinical applications. Eur. Heart J. 2006;27:2588-2605.

[40] Wang X, Du Y, Fan L, et al. Relationships between HDL-C, hs-CRP, with central arterial stiffness in apparently healthy people undergoing a general health examination. PLoS ONE. 2013;8:e81778.

[41] Wang X, Ye P, Cao R, et al. Triglycerides are a predictive factor for arterial stiffness: a community-based 4.8-year prospective study. Lipids Health Dis. 2016;15:97.

[42] Sever PS, Dahlöf B, Poulter NR, et al. Prevention of coronary and stroke events with atorvastatin in hypertensive patients who have average or lower-than-average cholesterol concentrations, in the Anglo-Scandinavian Cardiac Outcomes Trial- Lipid Lowering Arm (ASCOT-LLA): a multicentre randomised controlled trial. THE LANCET. 2003;361:10.

[43] Safar ME, Frohlich ED, editors. Atherosclerosis, Large Arteries and Cardiovascular Risk. Adv Cardiol. Basel. Karger. 2007; Vol 44:261-277. 
[44] Abedin M, Tintut Y, Demer LL. Vascular calcification: mechanisms and clinical ramifications. Arterioscler. Thromb. Vasc. Biol. 2004;24:1161-1170.

[45] Cominacini L, Rigoni A, Pasini AF, et al. The Binding of oxidized low density lipoprotein (oxLDL) to ox-LDL receptor-1 reduces the intracellular concentration of nitric oxide in endothelial Cells through an increased production of superoxide. J. Biol. Chem. 2001;276:13750-13755.

[46] Vita JA, Treasure CB, Nabel EG, et al. Coronary vasomotor response to acetylcholine relates to risk factors for coronary artery disease. Circulation. 1990;81:491-497.

[47] van der Harst P, Wagenaar LJ, Buikema H, et al. Effect of intensive versus moderate lipid lowering on endothelial function and vascular responsiveness to angiotensin II in stable coronary artery disease. Am. J. Cardiol. 2005;96:1361-1364.

[48] Bruno RM, Grassi G, Seravalle G, et al. Age- and sex-specific reference values for media/lumen ratio in small arteries and relationship with risk factors. Hypertension. 2018;71:1193-1200.

[49] McDonald's Blood Flow in Arteries, Sixth Edition: Theoretical, Experimental and Clinical Principles. Florida (FL). CRC Press. 2011.

[50] Wilkinson IB, Prasad K, Hall IR, et al. Increased central pulse pressure and augmentation index in subjects with hypercholesterolemia. J. Am. Coll. Cardiol. 2002;39:1005-1011.

[51] Janner JH, Godtfredsen NS, Ladelund S, et al. The association between aortic augmentation index and cardiovascular risk factors in a large unselected population. J. Hum. Hypertens. 2012;26:476-484.

[52] Leffler CW, Parfenova H, Jaggar JH. Carbon monoxide as an endogenous vascular modulator. Am. J. Physiol. Heart Circ. Physiol. 2011;301:H1-H11. 


\section{FIGURE LEGENDS}

Figure 1. Supine aortic systolic (A) and diastolic (B) blood pressure, and aortic pulse pressure (C) and augmentation index (D) in age- and sex-adjusted quartiles (Q1-Q4) of LDL-cholesterol during 5minute recordings. Q1 ( $\mathrm{n}=153), \mathrm{Q} 2(\mathrm{n}=158), \mathrm{Q} 3(\mathrm{n}=148)$ and Q4 $(\mathrm{n}=156)$; mean \pm standard error of the mean; ${ }^{*} p<0.05$, ANOVA for repeated measurements.

Figure 2. Heart rate (A), cardiac index (B), systemic vascular resistance index (C), and pulse wave velocity (D) in age- and sex-adjusted quartiles of LDL- cholesterol during 5-minute recordings. Quartiles as in Figure 1; mean \pm standard error of the mean; * $p<0.05$, ANOVA for repeated measurements. 
Figure 1.
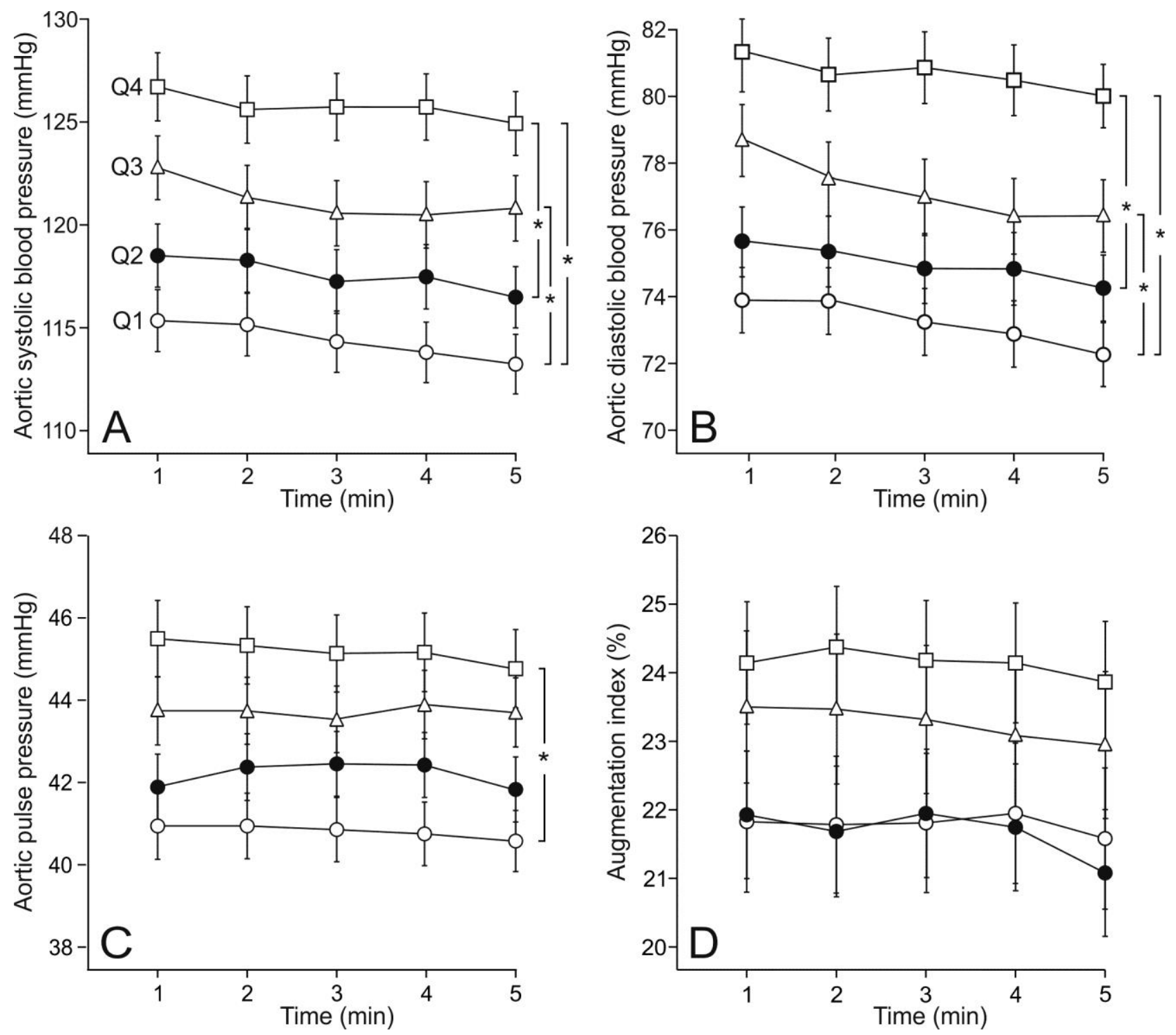
Figure 2.
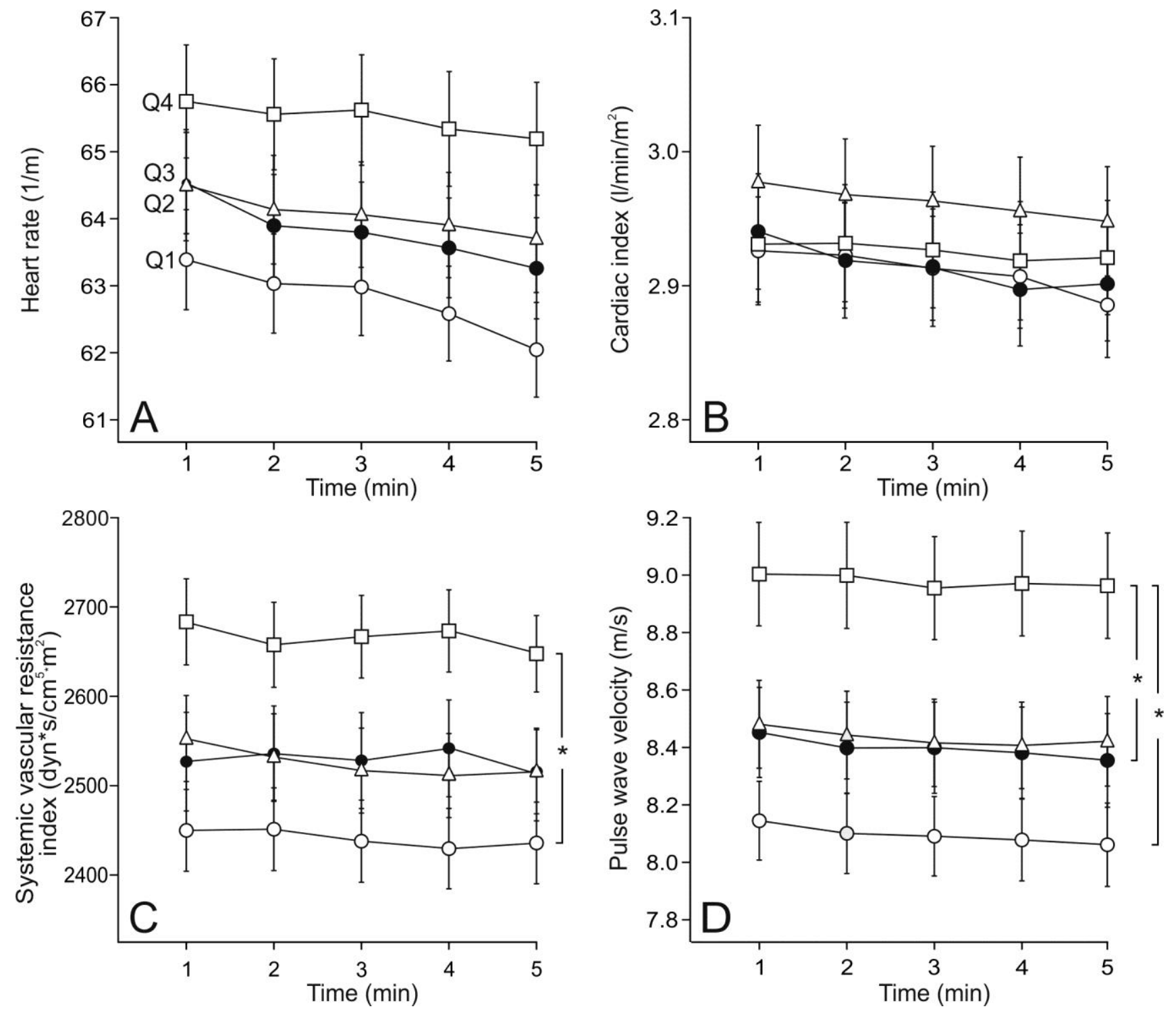
TABLE 1. Age and sex adjusted characteristics of the study population in quartiles of LDL cholesterol.

\begin{tabular}{|c|c|c|c|c|c|}
\hline & Overall & Q1 & $\mathrm{Q} 2$ & Q3 & Q4 \\
\hline Number & 615 & 153 & 158 & 148 & 156 \\
\hline Age (years) & $44.9(11.9)$ & $44.6(11.8)$ & $44.1(12.6)$ & $45.1(12.6)$ & $45.7(10.7)$ \\
\hline$B M I\left(k g / m^{2}\right)$ & $26.8(4.4)$ & $25.6(4.5)$ & $26.4(4.5)$ & $27.2(4.1)^{*}$ & $28.1(4.2)^{* \dagger}$ \\
\hline Alcohol (standard doses/week) & $4.5(5.7)$ & $3.8(4.9)$ & $4.1(5.1)$ & $4.9(6.5)$ & $5.0(6.3)$ \\
\hline Smokers (number / percentage) & $70 / 11.4 \%$ & $14 / 9.1 \%$ & $16 / 10.1 \%$ & $21 / 14.2 \%$ & $25 / 16.0 \%$ \\
\hline Systolic & $140.5(21.1)$ & $134.7(19.9)$ & $138.8(20.9)$ & $139.4(19.8)^{*}$ & $149.0(20.9)^{* \dagger}$ \\
\hline Diastolic & $89.3(12.5)$ & $85.3(12.0)$ & $88.6(12.9)$ & $90.0(11.4)^{*}$ & $94.0(12.1)^{* \dagger}$ \\
\hline eGFR $\left(\mathrm{ml} / \mathrm{min}\right.$ per $\left.1.73 \mathrm{~m}^{2}\right)$ & $98.8(18.1)$ & $102.0(18.1)$ & $101.3(18.3)$ & $97.7(17.1)$ & $94.2(18.0)^{* \dagger}$ \\
\hline QUICKI & $0.358(0.042)$ & $0.369(0.041)$ & $0.365(0.056)$ & $0.349(0.032)^{* \dagger}$ & $0.349(0.032)^{* \dagger}$ \\
\hline \multicolumn{6}{|l|}{ Fasting plasma } \\
\hline Creatinine $(\mu \mathrm{mol} / \mathrm{l})$ & $73.7(13.5)$ & $72.2(12.3)$ & $74.8(14.1)$ & $74.1(13.5)$ & $73.6(13.9)$ \\
\hline$C R P(m g / l)$ & $1.7(2.9)$ & $1.6(3.0)$ & $1.7(4.1)$ & $1.5(1.5)$ & $2.0(2.2)$ \\
\hline Total cholesterol (mmol/l) & $5.15(1.02)$ & $4.23(0.68)$ & $4.78(0.64)^{*}$ & $5.33(0.63)^{* \dagger}$ & $6.25(0.85)^{* \dagger \#}$ \\
\hline Triglycerides (mmol/l) & $1.23(0.76)$ & $1.05(0.96)$ & $1.17(0.69)$ & $1.23(0.62)$ & $1.49(0.70)^{* \dagger \#}$ \\
\hline$H D L-C(\mathrm{mmol} / \mathrm{l})$ & $1.58(0.44)$ & $1.71(0.488)$ & $1.54(0.41)^{*}$ & $1.56(0.41)^{*}$ & $1.52(0.43)^{*}$ \\
\hline LDL-C cholesterol (mmol/l) & $3.05(0.95)$ & $2.05(0.51)$ & $2.75(0.48)^{*}$ & $3.26(0.50)^{* \dagger}$ & $4.15(0.75)^{* \dagger \#}$ \\
\hline Insulin (mU/L) & $8.9(17.0)$ & $7.2(5.6)$ & $7.9(6.1)$ & $11.6(33.3)$ & $9.1(5.4)$ \\
\hline Glucose (mmol/l) & $5.44(0.58)$ & $5.34(0.69)$ & $5.40(0.59)$ & $5.49(0.48)$ & $5.55(0.54)^{*}$ \\
\hline
\end{tabular}

Mean (standard deviation), * $p<0.05$ vs Q1; ${ }^{\dagger} p<0.05$ vsQ2; ${ }^{*} p<0.05$ vs Q3; BMI, body mass index; eGFR, cystatin C based CDK-EPI formula for estimated glomerular filtration rate [31]; QUICKI, quantitative insulin sensitivity check index [30]; CRP, C-reactive protein; HDL-C, high density lipoprotein cholesterol; LDL-C, low density lipoprotein cholesterol. 
TABLE 2. Explanatory factors for haemodynamic variables in linear regression analyses with stepwise elimination.

\begin{tabular}{|c|c|c|c|c|c|c|c|c|c|}
\hline \multicolumn{4}{|c}{ Aortic systolic blood pressure: model 1 } & \multicolumn{4}{c|}{ Aortic systolic blood pressure: model 2 } \\
\hline & B & Beta & R squared & $p$ & & B & Beta & R squared & $p$ \\
\hline (Constant) & -16.615 & & & 0.738 & (Constant) & -98.362 & & 0.044 \\
\hline eGFR & -0.222 & -0.208 & 0.205 & $<0.001$ & PWV & 77.269 & 0.377 & 0.306 \\
\hline LDL-C & 3.343 & 0.165 & 0.259 & $<0.001$ & eGFR & -0.187 & -0.175 & 0.352 & $<0.001$ \\
\hline Age & 0.332 & 0.204 & 0.285 & $<0.001$ & LDL-C & 2.586 & 0.128 & 0.366 \\
\hline BMI & 0.531 & 0.122 & 0.304 & 0.003 & Sodium & 1.077 & 0.109 & 0.374 & 0.001 \\
\hline Sodium & 0.945 & 0.096 & 0.312 & 0.006 & QUICKI & -34.232 & -0.076 & 0.380 & 0.036 \\
\hline QUICKI & -38.761 & -0.086 & 0.317 & 0.026 & HDL-C & 4.509 & 0.103 & 0.384 & 0.005 \\
\hline & & & & & BMI & 0.434 & 0.099 & 0.389 & 0.014 \\
\hline
\end{tabular}

\begin{tabular}{|c|c|c|c|c|c|c|c|c|c|}
\hline \multicolumn{4}{|c|}{ Aortic diastolic blood pressure: model 1 } & \multicolumn{4}{c|}{ Aortic diastolic blood pressure: model 2 } \\
\hline & B & Beta & R squared & $p$ & & B & Beta & R squared & $p$ \\
\hline (Constant) & 19.283 & & & 0.588 & (Constant) & -32.811 & & 0.316 \\
\hline eGFR & -0.204 & -0.286 & 0.170 & $<0.001$ & PWV & 54.974 & 0.401 & 0.244 \\
\hline LDL-C & 2.265 & 0.168 & 0.217 & $<0.001$ & eGFR & -0.155 & -0.218 & 0.284 & $<0.001$ \\
\hline QUICKI & -33.714 & -0.112 & 0.238 & 0.005 & QUICKI & -35.114 & -0.116 & 0.306 & 0.001 \\
\hline Sex & 2.564 & 0.099 & 0.252 & 0.008 & Alcohol amount & 0.198 & 0.088 & 0.317 & 0.011 \\
\hline BMI & 0.283 & 0.097 & 0.257 & 0.021 & Sodium & 0.628 & 0.095 & 0.324 & 0.005 \\
\hline Sodium & 0.525 & 0.080 & 0.261 & 0.032 & Age & -0.149 & -0.137 & 0.330 & 0.006 \\
\hline & & & & & LDL-C & 1.466 & 0.109 & 0.337 & 0.006 \\
\hline
\end{tabular}

\begin{tabular}{|c|c|c|c|c|c|c|c|c|c|}
\hline \multicolumn{9}{|c|}{ Aortic pulse pressure: model 1 } & \multicolumn{5}{c|}{ Aortic pulse pressure: model 2 } \\
\hline & B & Beta & R squared & $p$ & & B & Beta & R squared & $p$ \\
\hline (Constant) & -46.757 & & & 0.092 & (Constant) & -50.479 & & 0.076 \\
\hline Age & 0.307 & 0.354 & 0.206 & $<0.001$ & Age & 0.190 & 0.219 & 0.206 & $<0.001$ \\
\hline BMI & 0.355 & 0.152 & 0.223 & $<0.001$ & PWV & 24.365 & 0.222 & 0.238 & $<0.001$ \\
\hline LDL-C & 1.331 & 0.123 & 0.230 & 0.003 & eGFR & -0.047 & -0.083 & 0.244 & 0.064 \\
\hline
\end{tabular}




\begin{tabular}{|l|l|l|l|l|l|l|l|l|l|l|l|}
\hline HDL-C & 2.398 & 0.102 & 0.235 & 0.012 & BMI & 0.291 & 0.125 & 0.248 & 0.003 \\
\hline Sodium & 0.417 & 0.079 & 0.240 & 0.032 & HDL-C & 2.677 & 0.114 \\
\hline & & & & & Sodium & 0.255 & 0.005 \\
\hline
\end{tabular}

\begin{tabular}{|c|c|c|c|c|c|c|c|c|}
\hline \multicolumn{9}{|c|}{ Augmentation index: model 1 } \\
\hline
\end{tabular}

\begin{tabular}{|c|c|c|c|c|c|c|c|c|c|}
\hline \multicolumn{5}{|c|}{ Pulse wave velocity: model 1} & \multicolumn{5}{|c|}{ Pulse wave velocity: model 2} \\
\hline & $\mathrm{B}$ & Beta & R squared & $p$ & & $\mathrm{~B}$ & Beta & R squared & $p$ \\
\hline (Constant) & 0.566 & & & $<0.001$ & (Constant) & 0.916 & & & $<0.001$ \\
\hline Age & 0.004 & 0.544 & 0.419 & $<0.001$ & Age & 0.004 & 0.501 & 0.419 & $<0.001$ \\
\hline Uric acid & 0.0002 & 0.172 & 0.493 & $<0.001$ & $\begin{array}{l}\text { Aortic systolic } \\
\text { blood pressure }\end{array}$ & 0.001 & 0.281 & 0.510 & $<0.001$ \\
\hline Triglycerides & 0.013 & 0.102 & 0.509 & 0.002 & Uric acid & 0.0002 & 0.154 & 0.555 & $<0.001$ \\
\hline LDL-C & 0.011 & 0.116 & 0.518 & 0.001 & Triglycerides & 0.012 & 0.095 & 0.568 & 0.003 \\
\hline Smoker & -0.023 & -0.081 & 0.525 & 0.005 & Smoker & -0.019 & -0.067 & 0.571 & 0.013 \\
\hline \multirow[t]{2}{*}{ BMI } & 0.002 & 0.080 & 0.528 & 0.019 & HDL-C & -0.019 & -0.088 & 0.575 & 0.006 \\
\hline & & & & & Sodium & -0.003 & -0.056 & 0.578 & 0.045 \\
\hline
\end{tabular}




\begin{tabular}{|c|c|c|c|c|}
\hline \multicolumn{5}{|c|}{ Systemic vascular resistance index: model 1 } \\
\hline & B & Beta & R squared & $p$ \\
\hline (Constant) & 3417.956 & & & $<0.001$ \\
\hline eGFR & -5.613 & -0.172 & 0.106 & $<0.001$ \\
\hline Weight & 9.149 & 0.246 & 0.142 & $<0.001$ \\
\hline Age & 5.122 & 0.103 & 0.159 & 0.031 \\
\hline Smoker & -220.835 & -0.123 & 0.171 & 0.001 \\
\hline Height & -8.445 & -0.133 & 0.181 & 0.005 \\
\hline LDL-C & 64.294 & 0.104 & 0.188 & 0.016 \\
\hline
\end{tabular}

For all these analyses, the skewed distribution of pulse wave velocity (PWV) was Lg10 transformed.

Variables in model 1: age, sex, body mass index (BMI) (for systemic vascular resistance BMI was replaced by height and weight), smoking status, consumption of standard drinks of alcohol per week, quantitative insulin sensitivity check index (QUICKI) [30], plasma C-reactive protein (CRP), sodium, uric acid, high density lipoprotein cholesterol (HDL-C), low density lipoprotein cholesterol (LDL-C), triglycerides, and cystatin C based CDK-EPI formula for estimated glomerular filtration rate (eGFR) [31].

Variables in model 2: model 1 + PWV; for augmentation index: model 1 + heart rate, systemic vascular resistance index (SVRI), and PWV; for PWV: model $1+$ aortic systolic blood pressure. 